\title{
Hawking radiation without black hole entropy
}

\author{
Matt Visser \\ Physics Department, Washington University, Saint Louis, Missouri 63130-4899
}

(2 December 1997; gr-qc/9712016)

\begin{abstract}
In this Letter I point out that Hawking radiation is a purely kinematic effect that is generic to Lorentzian geometries. Hawking radiation arises for any test field on any Lorentzian geometry containing an event horizon regardless of whether or not the Lorentzian geometry satisfies the dynamical Einstein equations of general relativity. On the other hand, the classical laws of black hole mechanics are intrinsically linked to the Einstein equations of general relativity (or their perturbative extension into either semiclassical quantum gravity or string-inspired scenarios). In particular, the laws of black hole thermodynamics, and the identification of the entropy of a black hole with its area, are inextricably linked with the dynamical equations satisfied by the Lorentzian geometry: entropy is proportional to area (plus corrections) if and only if the dynamical equations are the Einstein equations (plus corrections). It is quite possible to have Hawking radiation occur in physical situations in which the laws of black hole mechanics do not apply, and in situations in which the notion of black hole entropy does not even make any sense. This observation has important implications for any derivation of black hole entropy that seeks to deduce black hole entropy from the Hawking radiation.
\end{abstract}

Introduction: In Einstein gravity (general relativity), and in theories that perturbatively reduce to Einstein gravity, the notion of black hole entropy [1,2] is inextricably tied up with the existence of the Hawking radiation phenomenon 3, 19. Historically the notions were developed contemporaneously, and served to reinforce one another. The laws of black hole mechanics were developed first [5], with the formal similarity between the second law of black hole mechanics and the second law of thermodynamics then serving to suggest that black holes could be assigned an entropy [1,5]. But it was not until after the discovery of the Hawking radiation phenomenon [3, 1 , that the notion of black hole entropy became widely accepted, the laws of black hole mechanics then being promoted to the laws of black hole thermodynamics [2.,6].

However, with hindsight it is now possible to look back and realise that these two notions are actually rather distinct in their genesis, and that there are physical situations (not Einstein gravity) in which the two notions can be completely divorced - so that Hawking radiation can occur even in situations where the very notion of black hole entropy is meaningless. (For example, as I shall argue below, the acoustic black holes of Unruh [7], the solid-state black holes of Reznik [8], and the lattice black holes of Corley and Jacobson [9]. See the discussion in 10].)

In this Letter I emphasize that Hawking radiation is a purely kinematic phenomenon: It occurs in generic
Lorentzian geometries containing event horizons whenever one introduces a test field that propagates in an (approximate) Lorentz invariant manner. This should in fact have been realized immediately from the fact that Hawking's original derivation [3,4] makes no use of the Einstein equations. However, one would never think to even ask this question until after the advent of physical models of Lorentzian geometry that are distinct from Einstein gravity. The fact that sound waves in a flowing fluid couple to an acoustic metric that defines a Lorentzian geometry completely unconnected with the propagation of light is the best known example of such a physical system [7,10 16]. A clear pedagogical presentation of the notions of ergosphere, apparent horizon, event horizon, "surface gravity" and "acoustic black hole" in the acoustic model of Lorentzian geometry is presented in [10]. In fact, it is now known that the Hawking radiation process is sufficiently robust that approximate low-energy Lorentz invariance is quite sufficient to guarantee a thermal spectrum (subject to greybody distortion factors) 8, 9, 11, 14, 15, $17,23$.

On the other hand, black hole entropy, and in fact all of black hole thermodynamics and the classical laws of black hole mechanics, are intrinsically dynamical phenomena in that they depend critically on the perturbative validity of the Einstein equations. This can be seen from the modern derivations of the various laws of black hole mechanics [5. 60, which proceed by explicitly invoking the Einstein equations together with the various classical energy conditions of Einstein gravity [6,24,25]. (This fact is somewhat obscured in some of the early discussions of black hole mechanics where consideration is implicitly limited to the standard Schwarzschild, ReissnerNordström, Kerr, and Kerr-Newman black holes.) That the laws of black hole mechanics generally fail for the acoustic black holes of the acoustic Lorentzian geometries is explicitly pointed out in [10].

The impact of these results is perhaps a little subtle: The existence of the Hawking flux in any candidate theory of quantum gravity is not itself a test of any dynamical aspect of quantum gravity. The existence of the Hawking flux is not even a test of the dynamics of the lowenergy effective theory. Instead, the Hawking flux tests the extent to which the candidate theory of quantum gravity is capable of reproducing the Lorentzian manifold structure that we have by now come to believe is an inescapable part of the kinematics of any phenomenologically acceptable theory of gravity (at least in the lowenergy limit probed by current experiments [26]).

It is only after one imposes (or derives) dynamical 
equations for the low energy effective theory, and only provided that these low-energy dynamical equations are the Einstein equations (possibly plus higher-order corrections), that we can invoke the laws of black hole thermodynamics to see that black holes can be assigned entropies proportional to their area (possibly plus higherorder corrections) 27 31. Thus a calculation of the Hawking flux, in any candidate theory of quantum gravity, supports the notion of black hole entropy only insofar as it provides reasons for believing the perturbative applicability of the dynamics encoded in the Einstein equations.

These conclusions hold independently of whatever model one wishes to propose for quantum gravity, as they require knowledge only of the low-energy sub-Planckian phenomenology - where we at least think we understand the basic issues.

Acoustic Lorentzian geometries: The acoustic model for Lorentzian geometry is not widely-known outside of the confines of the general relativity community so I shall provide a brief description here. The model arises from asking the deceptively simple question of how sound waves propagate in a flowing fluid. Under suitable restrictions (vorticity-free flow, barotropic equation of state, zero viscosity) it can be shown that linearizing the combined Euler and continuity equations of non-relativistic fluid mechanics leads to sound waves (phonons) that are described by a scalar field. This phonon is a massless scalar field that is minimally coupled to the "acoustic metric" [7, 10,13, 15]. The acoustic metric is an algebraic function of the density, speed of sound, and velocity of the flowing fluid explicitly given by

$$
g_{\mu \nu}(t, \vec{x}) \equiv \frac{\rho}{c}\left[\begin{array}{ccc}
-\left(c^{2}-v^{2}\right) & \vdots & -\vec{v} \\
\cdots \cdots \cdots+\cdots & \cdot & \cdots \cdots \\
-\vec{v} & \vdots & I
\end{array}\right] .
$$

(Here $I$ is the $3 \times 3$ identity matrix.) The equation of motion for the phonon field is simply the usual d'Alembertian equation [7, 10,13,15]

$$
\Delta \psi \equiv \frac{1}{\sqrt{-g}} \partial_{\mu}\left(\sqrt{-g} g^{\mu \nu} \partial_{\nu} \psi\right)=0 .
$$

This model is sufficiently rich to enable probing of almost all of the kinematic aspects of general relativity (the existence of a Lorentzian geometry), without the dynamics (the Einstein equations). The dynamics of the acoustic Lorentzian geometry are of course governed by the ordinary nonrelativistic Euler and continuity equations. (The acoustic Lorentz geometries are not completely arbitrary in that they automatically satisfy the stable causality condition 10,13], which thereby precludes some of the more entertaining causality related problems that can arise in Einstein gravity.) Nevertheless, the acoustic Lorentz geometries are sufficiently general so as to contain ergospheres, trapped regions, apparent horizons, event horizons (absolute horizons), and the full panoply of technical machinery for the kinematic aspects of black hole physics [10]. Black holes are defined as regions from which phonons (which are represented by null geodesics of the acoustic metric) cannot escape - because the fluid is flowing inward at greater than the local speed of sound. At the (future) event horizon the normal component of the fluid velocity is inward pointing and equals the local speed of sound, $v_{\perp}=c$. The notion of "surface gravity" can be defined as for general relativistic black holes; and for stationary flows measures the extent to which the natural time parameter defined by the timelike Killing vector fails to be an affine parameter for those null geodesics that just skim the event horizon. The "surface gravity" can be calculated to be [10]

$$
g_{H}=\frac{1}{2} \frac{\partial\left(c^{2}-v_{\perp}^{2}\right)}{\partial n}=c \frac{\partial\left(c-v_{\perp}\right)}{\partial n} .
$$

This generalizes the result of Unruh [7, 15] to the case where the speed of sound is position dependent and/or the acoustic horizon is not the null surface of the time translation Killing vector. This result is also compatible with that deduced for the solid-state black holes of Reznik [8], the lattice black holes of Corley and Jacobson [9], and with the "dirty black holes" of [32]. In the special case where the speed of sound is independent of position, and the fluid impinges on the event horizon at right angles, (e.g. if the geometry is static rather than just stationary) the surface gravity is identical to the ordinary three-dimensional acceleration of the fluid as it crosses the horizon [10].

As originally discussed by Unruh [7], (and subsequent papers 11.14 231) an acoustic event horizon will emit Hawking radiation in the form of a thermal bath of phonons at a temperature

$$
k T_{H}=\frac{\hbar g_{H}}{2 \pi c} .
$$

(Yes, this really is the speed of sound in the above equation, and $g_{H}$ is really normalized to have the dimensions of a physical acceleration.) This result also applies, with suitable modifications, to the solid-state black holes of Reznik 8] and the lattice black holes of Corley and Jacobson [9]. Using the numerical expression

$$
T_{H}=\left(1.2 \times 10^{-9} \mathrm{Km}\right)\left[\frac{c}{1000 m s^{-1}}\right]\left[\frac{1}{c} \frac{\partial\left(c-v_{\perp}\right)}{\partial n}\right],
$$

it is clear that experimental verification of this acoustic Hawking effect will be rather difficult. (Though, as Unruh has pointed out [7], this is certainly technologically easier than building [general relativistic] microblack holes in the laboratory.)

Despite the technological difficulties inherent in bringing these acoustic black holes to experimental realization, they already provide us with a clean theoretical laboratory that sharply divorces the kinematic aspects of general relativity (Lorentzian geometry) from the dynamic 
aspects (the Einstein equations). That such a divorce is even possible in physically realisable systems was not clear before the advent of the acoustic Lorentzian geometries.

Now that we have at least one clean theoretical laboratory that makes this separation, theorists can calmly take the next step and even divorce themselves from the underlying fluid mechanics - now turning interest to Lorentzian geometries in general without making any commitment to any particular geometrodynamics, be it Einstein geometrodynamics or Euler geometrodynamics (acoustic geometrodynamics). Once this critical conceptual step is made, it is clear that the calculations of $17,9,11,14,15,17,23$, though they were inspired by the acoustic model, actually prove that Hawking radiation is a completely kinematic effect independent of any underlying dynamics for the Lorentzian geometry [10].

Black hole mechanics: The dynamical origin of the laws of black hole mechanics is evident from the fact that the various proofs in the literature explicitly use either the Einstein equations plus the energy conditions [5, 6, 24, 25], or at an absolute minimum, the existence of a diffeomorphisim invariant Lagrangian governing the evolution of the Lorentzian geometry [27,28]. For instance:

Zeroth law: In Einstein gravity the constancy of the surface gravity (mutatis mutandis the Hawking temperature) over the event horizon of a stationary black hole (with Killing horizon) follows from the Einstein equations plus the Dominant Energy Condition [6, pages 331-334]. In the acoustic model it is not even necessary for the event horizon of a stationary acoustic black hole to be a Killing horizon [10], and with sufficiently complicated fluid flows one can set up arbitrarily complicated patterns of "surface gravity". In general relativity the fact that stationary but non-static black holes are axisymmetric is deduced from the fact that non-axisymmetric black holes are expected to loose energy via gravitational radiation and so dynamically relax to an axisymmetric configuration - in the fluid dynamic models there is no particular reason to even consider dynamically relaxation since the flow can be maintained by external forces. With no Einstein equations, no energy conditions, and not even the guarantee of a Killing horizon, there is no zeroth law [10].

First law: The most general derivation of the first law of black hole mechanics still requires a dynamical evolution for the Lorentzian geometry that is governed by a diffeomorphisim invariant Lagrangian [27,28. Subject to this restriction the entropy of a black hole can be defined in terms of the Lagrangian governing the dynamics by 27,31

$$
S=k_{B} \int_{H} \frac{\delta \mathcal{L}}{\delta R_{\mu \nu \sigma \rho}} \epsilon_{\nu \nu} \epsilon_{\sigma \rho} \sqrt{{ }^{2} g} d^{2} x .
$$

The integral runs over some suitable cross-section of the horizon. If the Lagrangian is Einstein-Hilbert (possibly plus corrections), then the entropy will be proportional to the area (plus corrections) 27 31. In the absence of a covariant dynamics, it does not even make sense to assign an entropy to the event horizon.

Second law: Proofs of the second law of black hole mechanics, the Hawking area increase theorem, explicitly invoke the Einstein equations plus the null energy condition 6,24. Proofs of the generalized second law of black hole thermodynamics (the increase of total entropy, ordinary entropy plus black hole entropy) implicitly invoke covariant dynamics arising from a diffeomorphisim invariant Lagrangian (via appeal to the first law) [33 35], and sometimes make even more specific model-dependent assumptions about the matter fields 35.

Third law: The third law of black hole mechanics (the impossibility of reaching extremality), is again intrinsically dynamical. There is considerable ambiguity on how to precisely formulate the third law (Nernst theorem), and I shall direct interested readers to the recent paper by Wald [36.

Semiclassical quantum gravity: With this build up, it is now clear that semiclassical quantum gravity will always be well behaved with regard to the issues raised in this Letter. Because semiclassical quantum gravity still models the universe by a Lorentzian spacetime, there will still be Hawking radiation from any event horizon. Because semiclassical quantum gravity has an effective action that is the Einstein-Hilbert term plus higher-curvature corrections, the black hole entropy will be proportional to the area plus corrections. Since any putative model for quantum gravity must reduce to semiclassical quantum gravity in the sub-Planckian limit, the calculation of emission rates and entropies in candidate models of quantum gravity are excellent consistency checks that these models must satisfy.

If one has a complicated model for quantum gravity, then the complicated calculations required to verify these non-trivial low-energy consistency checks may indeed seem miraculous - but the miracle is more a reflection of the complexity of the candidate model for quantum gravity than it is any guarantee of the physical correctness of the model.

String-inspired scenarios: The quantum gravity models most ardently being pursued at this stage are the various string-inspired scenarios. Recent progress in calculating the Hawking flux in these string-inspired models, including (in some limits) greybody factors, and progress in constructing a microphysical statistical mechanics analog for the black hole entropy has generated much excitement. See, for instance, 3742 . Most intriguingly, many of these results seem to be largely independent of the technical details of fundamental string theory [39. From the point of view argued in this Letter, this is only natural: By showing that in certain limits stringinspired models produce a Hawking flux one is verifying that in these limits the string models are compatible with the existence of a Lorentzian geometry with event horizon. By calculating the precise spectra of the stringmodel Hawking fluxes (greybody factors), and compar- 
ing them with the greybody factors for the canonical black holes (Schwarzschild, Reissner-Nordström, Kerr, Kerr-Newman) one is testing the extent to which stringinspired models reproduce standard physics. In fact, calculating greybody factors is a complicated way of implicitly checking that the low-energy dynamics is in fact Einstein-Hilbert. (A warning: since we expect the lowenergy limit of string models to reduce to Einstein gravity plus stringy corrections, we should expect stringy black holes to be canonical black holes plus stringy corrections, and so we should not expect the greybody factors to be identical to the canonical ones beyond lowest nontrivial order in the string tension.)

Thus I argue that calculations of greybody factors in string-inspired models for quantum gravity are best viewed as non-trivial consistency checks on the ability of these models to be compatible with a suitable low-energy semiclassical quantum gravity. These consistency checks are not unique to the string-inspired models and must be faced by any candidate for quantum gravity. The feature of the string-inspired models that is more important than the precise form of the greybody factors is the fact that these scenarios appear to provide a unitary description of the Hawking flux.

Turning to the black hole entropy: Since we believe that the low-energy limit of the string models are pointparticle field theories defined on Lorentzian geometries, and that in this limit the string models reproduce Einstein gravity, then we automatically know (without further calculation) that black holes can be assigned an entropy proportional to their area. The feature of the string-inspired models that goes beyond semiclassical quantum gravity is the fact that this notion of entropy can be continuously extended to regions of parameter space where black holes do not exist and explicit state counting calculations hold sway.

Acknowledgements: I wish to thank Ted Jacobson for helpful comments. This research was supported by the US Department of Energy.

[1] J.D. Bekenstein, "Black holes and entropy", Phys. Rev. D 7, 2333-2346 (1973).

[2] J.D. Bekenstein, "Generalized second law of thermodynamics in black hole physics", Phys. Rev. D 9, 3292-3300 (1974).

[3] S.W. Hawking, "Black hole explosions?", Nature 248, 30-31 (1974).

[4] S.W. Hawking, "Particle creation by black holes", Commun. Math. Phys. 43 199-220 (1975).

[5] J.M. Bardeen, B. Carter, and S.W. Hawking, "The four laws of black hole mechanics", Commun. Math. Phys. 31 161-170 (1973).

[6] R.M. Wald, General relativity, (University of Chicago,
Chicago, 1984). See especially pages $330-337$, an pages 399-418.

[7] W. G. Unruh, "Experimental black hole evaporation?", Phys. Rev. Lett, 46, 1351-1353 (1981).

[8] B. Reznik, "Origin of the thermal radiation in a solidstate analog of a black hole", gr-qc/9703076.

[9] S. Corley and T. Jacobson, "Lattice black holes", hepth/9709166.

[10] M. Visser, "Acoustic black holes: horizons, ergospheres, and Hawking radiation", gr-qc/9712010.

[11] T. Jacobson, "Black hole evaporation and ultrashort distances", Phys. Rev. D 44, 1731-1739 (1991).

[12] G. Comer, "Superfluid analog of the Davies-Unruh effect", August 1992, (unpublished).

[13] M. Visser, "Acoustic propagation in fluids: an unexpected example of Lorentzian geometry", gr-qc/9311028.

[14] T. Jacobson, "Black hole radiation in the presence of a short distance cutoff", Phys. Rev. D 48 728-741 (1993), hep-th/9303103.

[15] W.G. Unruh, "Sonic analogue of black holes and the effects of high frequencies on black hole evaporation", Phys. Rev. D 51, 2827-2838 (1995);

E-printed as "Dumb holes and the effects of high frequencies on black hole evaporation", gr-qc/9409008.

[16] D. Hochberg, "Evaporating black holes and collapsing bubbles in fluids", March 1997, (unpublished).

[17] R. Brout, S. Massar, R. Parentani, Ph. Spindel, "Hawking radiation without transplanckian frequencies", Phys. Rev. D 52, 4559-4568 (1995); hep-th/9506121.

[18] T. Jacobson, "Introduction to black hole microscopy", published in Mexican School on Gravitation 1994, pages 87-114, hep-th/9510026.

[19] T. Jacobson, "On the origin of the outgoing black hole modes", Phys. Rev. D 53, 7082-7088 (1996), hepth/9601064.

[20] S. Corley and T. Jacobson, "Hawking spectrum and high frequency dispersion", Phys. Rev. D 54 1568-1586 (1996), hep-th/9601073.

[21] S. Corley, "Particle creation via high frequency dispersion", Phys. Rev. D 55 6155-6161 (1997).

[22] S. Corley, "Computing the spectrum of black hole radiation in the presence of high-frequency dispersion: an analytical approach", hep-th/9710075.

[23] B. Reznik, "Trans-Planckian tail in a theory with a cutoff", Phys. Rev. D 55, 2152-2158 (1997); gr-qc/9606083.

[24] S. W. Hawking and G. F. R. Ellis, The large scale structure of space-time, (Cambridge University Press, England, 1973).

[25] M. Visser, Lorentzian Wormholes - from Einstein to Hawking, (American Institute of Physics, New York, 1995).

[26] C.M. Will, "Theory and experiment in gravitational physics", revised edition, (Cambridge University Press, England, 1993).

[27] R.M. Wald, "Black Hole Entropy is Noether Charge", Phys. Rev. D 48, 3427-3431 (1993); gr-qc/9307038.

[28] V. Iyer and R.M. Wald, "Some properties of the Noether charge and a proposal for dynamical black hole entropy", Phys. Rev. D 50, 846-864.

[29] M. Visser, "Dirty black holes: entropy versus area", 
Phys. Rev. D 48, 583-591 (1993); hep-th/9303029.

[30] M. Visser, "Dirty black holes: entropy as a surface term", Phys. Rev. D 48, 5697-5705 (1993); hep-th/9307194

[31] T. Jacobson, G. Kang, R.C. Myers, "On black hole entropy", Phys. Rev. D 49, 6587-6598 (1994); grqc/9312023.

[32] M. Visser, Dirty black holes: thermodynamics and horizon structure, Phys. Rev. D. 46, 2445-2451 (1992); hepth/9203057.

[33] V.P. Frolov and D.N. Page, "Proof of the generalized second law for quasitationary semiclassical black holes", Phys. Rev. Lett. 71, 3902-3905 (1993).

[34] T. Jacobson, G. Kang, R.C. Myers, "Increase of Black Hole Entropy in Higher Curvature Gravity", Phys. Rev. D 52, 3518-3528 (1995).

[35] S. Mukohyama, "New proof of the generalized second law", Phys. Rev. D 56, 2192-2201 (1997).

[36] R.M. Wald, "The 'Nernst Theorem' and Black Hole Thermodynamics", Phys. Rev. D 56, 6467-6474 (1997); gr-qc/9704008.

[37] G. Horowitz and J. Polchinski, "A Correspondence Principle for Black Holes and Strings", Phys. Rev. D 55, 6189 (1997); hep-th/9612146.

[38] G. Horowitz, "Quantum states of Black holes", grqc/9704072, to appear in the proceedings of the symposium on Black Holes and Relativistic Stars, in honor of S. Chrandrasekhar, December 1996.

[39] J. Maldacena and A. Strominger, "Universal low-energy dynamics for rotating black holes", Phys. Rev. D 56, 4975-4983; hep-th/9702015.

[40] M. Cvetic and F. Larsen, "Greybody factors for rotating black holes in four dimensions", hep-th/9706071.

[41] S.S. Gubser, "Absorption of photons and fermions by black holes in four dimensions", hep-th/9706100.

[42] R. Emparan, "Absorption of scalars by extended objects", hep-th/9706204. 